\title{
Specific immunoglobulin E in nasal secretions for the diagnosis of local allergic rhinitis*
}

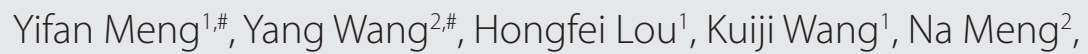 \\ Luo Zhang ${ }^{1,2,3}$, Chengshuo Wang' \\ ' Department of Otolaryngology Head and Neck Surgery, Beijing TongRen Hospital, Capital Medical University, Beijing 100730, Chin \\ 2Beijing Key Laboratory of nasal diseases, Beijing Institute of Otolaryngology, Beijing 100005, China \\ Department of Allergy, Beijing TongRen Hospital, Capital Medical University, Beijing 100730, China
}

Rhinology 57: 4, 313 - 320, 2019

https://doi.org/10.4193/Rhin18.292

*Received for publication:

December 2, 2018

Accepted: May 3, 2019

"These authors contributed equally to this study

\begin{abstract}
Background: The diagnostic value of serum specific Immunoglobulin E (slgE) and nasal allergen provocation test (NAPT) has been well investigated in local allergic rhinitis (LAR). We hypothesized that nasal local slgE could be used for the diagnosis of LAR instead of NAPT.
\end{abstract}

Methods: This was a prospective single center study. Overall, 212 chronic rhinitis patients were screened, of whom 73 were recruited based on negative findings for serum lgE and positive findings for local eosinophils. Ten healthy subjects were also recruited as controls. All participants completed questionnaires at recruitment to record their demographic data, nasal symptom severity, and physician-diagnosed comorbid asthma. Symptom severity was recorded using a visual analogue scale (VAS) of $10 \mathrm{~cm}$ and allergic status was assessed by serum slgE. Nasal secretions were collected for analysis of local slgE and eosinophils, and NAPT was performed for confirmation of LAR.

Results: Overall, 14 patients demonstrated positive local slgE results. Twelve of these patients had significantly higher local slgE levels compared to controls, and also demonstrated positive NAPT results. The VAS scores, nasal airway resistance measured by active rhinomanometry, and the levels of local slgE, ECP, histamine and leukotriene $C 4$ were significantly increased from baseline values following NAPT. Sensitivity, specificity, and diagnostic accuracy of local slgE for diagnosis of LAR were $91.7 \%$ respectively.

Conclusions: The measurement of local slgE levels in nasal secretion is a reliable and effective diagnostic method for LAR.

Keywords: diagnosis, local allergic rhinitis, nasal allergen provocation test, specific immunoglobulin $\mathrm{E}$

\section{Introduction}

Rhinitis is defined as an inflammation of the nasal mucosa, usually characterized by rhinorrhea, sneezing, nasal obstructive and itching ${ }^{(1)}$. Rhinitis is a common respiratory disease, which currently affects about $20-40 \%$ of the population in the western countries, and its incidence is still rising ${ }^{(2)}$. The adverse impact of rhinitis has not only been associated with chronic rhinosinusitis, asthma, psychological impairment and sleep impairment, but also with decreased study and work productivity ${ }^{(3-6)}$. Rhinitis has been traditionally classified as allergic rhinitis (AR) and nonal- lergic rhinitis, and the diagnosis has been based on the clinical history, endoscopic examination, skin prick test (SPT) or serum IgE levels to different kinds of sensitizing allergens ${ }^{(1)}$. However, with increasing focus on optimizing personal treatment in recent years, especially in the field of chronic upper airway diseases, the two broad phenotypes of AR and non-AR cannot meet the needs of precision medical, especially with more and more phenotypes of rhinitis being classified.

Huggins and colleagues ${ }^{(7)}$ first demonstrated that specific Immunoglobulin $E$ (slgE) antibodies were produced locally in AR 
patients with negative skin tests; and nearly thirty years later, based on similar findings Powe and colleagues ${ }^{(8)}$ defined this phenomenon as "entopy". Recently, Rondon and colleagues ${ }^{(9)}$ have defined this specific phenotype of rhinitis as "local allergic rhinitis" (LAR), which further more recent research has suggested that $L A R$ is an entity well differentiated from $A R^{(10)}$. Rondon and colleagues ${ }^{(10-12)}$ have further proposed that a diagnosis of LAR may be made based on several criteria; including 1) local production of IgE, 2) Th2 inflammatory pattern in nasal secretion when exposed to sensitizing allergens, and 3) positive results of nasal allergen provocation test (NAPT) without systemic atopy including increased level of slgE, tryptase and eosinophil cationic protein (ECP) in the nasal secretion. There is little doubt that NAPT is the most important method for the diagnosis of LAR, and that it is potentially important in the proper selection of an individualized therapeutic strategy in clinically complicated cases with sensitizations to various antigens ${ }^{(9,13-15)}$. However, NAPT also has several limitations in clinical practice; in particular the absence of standardized methods and some reagents, the wide variety of test protocols, need to be performed by trained personnel under close medical supervision and are difficult to perform on pediatric patients ${ }^{(16,17)}$.

We have recently demonstrated that the level of slgE levels in nasal secretion (local slgE) is an effective and reliable alternative to serum IgE for a diagnosis of allergic rhinitis ${ }^{(18)}$. Thus, we have hypothesized that the level of local slgE may also be effective, relatively simple and reliable alternative to NAPT for the diagnosis of LAR.

\section{Materials and methods}

\section{Study design and patients}

This prospective study was conducted from 1st February 2017 to 31 st August 2017, in a single center. Overall, 212 patients suspected to have rhinitis based on the presence of common symptoms of nasal obstruction, rhinorrhea, sneezing and itching were screened consecutively from the allergy-rhinology clinic of Beijing TongRen Hospital. Questionnaires were completed by each participant to record demographic data and nasal symptom severity; and diagnosis of AR was confirmed based on the presence of symptoms and clinically relevant serum slgE results. Similarly, physician-diagnosed comorbid asthma was assessed according to presence of symptoms and lung function tests. Nasal secretions were collected for analysis of local slgE and eosinophils, which were assessed according to the study by Howarth and colleagues ${ }^{(19)}$. Thus, to be included in the study, eligible patients were required to have findings for both negative serum IgE and positive local eosinophils in nasal secretions, and could therefore be classified as LAR or NARES patients. NAPT was performed on these patients; and setting the NAPT results as the golden standard, the value of local slgE in diagnosing LAR was evaluated.
Patients with any immunological disease, chronic rhinosinusitis and/or nasal polyposis, any respiratory infection in the previous 4 weeks were excluded, as were pregnant and lactating women. CRS patients were excluded based on history, nasal endoscopic examination and, findings from CT scans. Patients were also excluded if they had taken systemic corticosteroids over the past 3 months, intranasal corticosteroids over the past 4 weeks, antihistamines over the past 2 weeks, or vasoconstrictors over the past 1 week. Additionally, based on the diagnostic criteria of LAR, patients found to have positive serum slgE results and negative local eosinophils results were also excluded from the cohort. The study was conducted in full accordance with Declaration of Helsinki and approved by the Medical Ethics Committee of Beijing TongRen Hospital; and all patients provided written informed consent prior to entry into the study and collection of data.

\section{Visual analogue scale}

The severity of nasal symptoms; including nasal obstruction, anterior or posterior rhinorrhea (watery, mucous or purulent), sneezing and nasal/eye itching was recorded using a visual analogue scale (VAS) of $10 \mathrm{~cm}$. Each symptom was categorized as 'mild' (VAS: $0-3 \mathrm{~cm}$ ), 'moderate' (VAS: $>3-7 \mathrm{~cm}$ ), or 'severe' (VAS $>7 \mathrm{~cm})$.

\section{Rhinomanometry and nasal airway resistance}

Nasal airway resistance (NAR) was assessed at a transnasal pressure difference of $150 \mathrm{~Pa}$ by active rhinomanometry (ATMOS Medizin Technik GmbH\&Co., Feldkirch, Germany).

\section{Serum slgE}

Serum slgE levels to common aeroallergens were determined using a fluoroenzyme immunosorbent assay (UniCAP, Uppsala, Sweden); with a value for serum slgE $\geq 0.35 \mathrm{kU} / \mathrm{L}$ regarded as positive. The slgE examination was performed with a panel of allergens including Dermatophagoides farinae (Der f), Dermatophagoides pteronyssinus (Der p), Candida albicans, Mugwort, Penicillium notatum, Cladosporium, Alternaria, and Aspergillus.

\section{Local slgE}

Local slgE was determined in nasal secretions, which were collected and processed as described previously ${ }^{(20)}$. Briefly, 30 minutes before NAPT and immediately following NAPT when the patient experienced symptoms of irritation, nasal secretions were obtained by inserting a postoperative sinus sponge pack in each nostril for about 5 minutes. The quantity of secretions collected in each sponge was determined by comparing the weight of the sponge before and after insertion into the nasal cavity, and the secretion was extracted from the sponge by addition of $2 \mathrm{~mL}$ of $0.9 \%$ sodium chloride solution.

All sponges were stored at $4{ }^{\circ} \mathrm{C}$ for at least two hours and then transferred to a $5 \mathrm{~mL} \mathrm{BD}$ syringe. The bulk of the nasal secretion 
was forced out of the sponges using the piston of the syringe and centrifuged at $1500 \mathrm{~g}$ for 15 minutes at $4{ }^{\circ} \mathrm{C}$. The supernatants were separated and stored in aliquots at $-20^{\circ} \mathrm{C}$ until analysis for the presence of slgE. At the time of assay, an aliquot was also assessed to make sure there was no contamination by bacteria or fungi, or disturbance in the other factors. Local sigE in nasal secretions was assessed using the fluoroenzyme immunosorbent assay (UniCAP, Uppsala, Sweden) and the panel of aeroallergens as for serum. Again a value for $\operatorname{sig} E \geq 0.35 \mathrm{kU} / \mathrm{L}$ was regarded as positive.

\section{Nasal allergen provocation test}

Nasal allergen provocation test (NAPT) was performed according to the guidelines of European Academy of Allergy and Clinical Immunology ${ }^{(21)}$. The patients were first seated at room temperature for 30 minutes to minimize the effects of daily-life stimulation, before undergoing saline $(0.9 \% \mathrm{NaCl})$ challenge tests to exclude nasal hyperreactivity. After a 30-min adaptation period, the patients were challenged with saline, Der-f and mugwort solutions $(0.004 \mathrm{mg} / \mathrm{ml}, 0.04 \mathrm{mg} / \mathrm{ml}, 0.4 \mathrm{mg} / \mathrm{ml}$, and $4 \mathrm{mg} / \mathrm{ml}$, WOLWOPHARMA, Zhejiang, China), delivered to the inferior turbinate using a nebulizer with a fixed volume of $100 \mathrm{ul}$ per puff (the better patent side). Solutions were applied to both nostrils in increasing concentrations at 10 minutes intervals; with the subjects holding the breath after a deep inspiration to avoid allergen being inhaled into the lower airways; until the appearance of allergic symptoms. The clinical symptoms and the change in nasal airway resistance (NAR) were reassessed with active rhinomanometry after NAPT.

Total symptom scores represented the sum of the scores for: sneezing $(0-2$ sneezes $=0 ; 3-5=1 ;>5=2)$; rhinorrhea (moderate $=1$; severe $=2$ ); tearing+itching (eyes and/or throat $=1$; conjunctivitis+ cough+urticaria and/or dyspnea $=2)^{(22)}$. NAPT was considered positive if the total symptom score was $\geq 4$ or nasal airflow was reduced by $60 \%$ or more from the baseline level. Challenge was also considered positive if nasal airflow was reduced by $20 \%$ or more plus the symptom score was $\geq 3$. To avoid any stimulation of the nasal mucosa caused by postoperative sinus sponge pack in each nostril, the NAPT was performed at least $1 \mathrm{~h}$ after removing the sponge from the nostril.

\section{Measurement of inflammatory mediators}

Inflammatory mediators were measured in nasal secretions collected before and after NAPT, as described above for slgE. Commercial ELISA kits were used to measure the levels of total protein (Beyotime, Beijing, China); and the levels of eosinophil cationic protein (ECP) (CUSABIO, Wuhan, Hubei province, China), the levels of histamine (CUSABIO), and the levels of leukotriene C4 (R\&D Systems, Minneapolis, MN, USA). The level of each mediator was calculated as the concentration in $\mathrm{ng} / \mathrm{g}$ protein. When the level of a mediator measured was below the detecti-

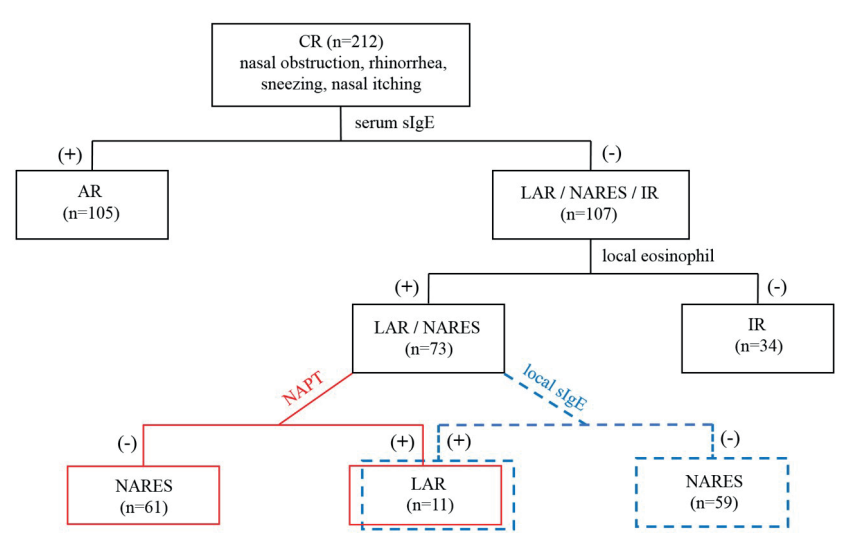

Figure 1. The stages for diagnosis of different chronic rhinitis endotypes. The red line (NAPT) indicates the conventional diagnostic stage (golden standard) and the blue dotted line (local slgE) indicates the newly proposed diagnostic step differentiation between LAR and NARES. AR, allergic rhinitis; $C R$, chronic rhinitis; IR, idiopathic rhinitis; LAR, local allergic rhinitis; NAPT, nasal allergen provocation test; NARES, nonallergic rhinitis with eosinophilia syndrome.

on level in the initial measurement, the results of that particular sample was excluded.

\section{Statistical analysis}

Setting the NAPT results as the golden standard, the sensitivity, specificity, positive predictive value (PPV), negative predictive value (NPV), and diagnostic accuracy (DA) of local slgE were determined for predicting the value of LAR. A true positive value was defined as the ability of local slgE to accurately predict the diagnosis of LAR, and a true negative value was defined as failing to diagnose LAR. The variables between the clusters were compared by one-way analysis of variance, Chi-square test and Tukey's test using SPSS version 22.0 (IBM Corp., Armonk, NY, USA) and GraphPad Prism 7.0 software (GraphPad Software, Inc., La Jolla, CA, USA). Data were expressed as median and interquartile range, unless otherwise specified. Statistical significance was set at a $p$-value $\leq 0.05$.

\section{Results}

Figure 1 shows the stages in the diagnosis of the different endotypes of chronic rhinitis in the present study; with the possibility of replacing NAPT by measurement of local slgE for differentiating between LAR and NARES. Overall, 212 patients with rhinitis symptoms (nasal obstruction, rhinorrhea, sneezing and nasal itching) were screened and 73 eligible patients ( 42 males and 31 females; age range 25 years to 54 years, mean $=38.0 \pm 12.4$ years) were further investigated. Similarly 10 healthy subjects were also investigated as controls. Of the eligible rhinitis patients, 14 had positive local slgE results and 2 patients concomitant asthma (Table 1). After performing the NAPT on these 73 rhinitis patients, 12 rhinitis patients $(7$ males and 5 females, mean $=$ 
Table 1. Demographic characteristics, NAPT and local slgE results of patients deemed to have LAR.

\begin{tabular}{|ccccccc}
\hline No. & Age & Gender & Asthma & NAPT & $\begin{array}{c}\text { Local } \\
\text { slgE }\end{array}$ \\
\hline 1 & 39 & $\mathrm{M}$ & $\mathrm{N}$ & + & + \\
\hline 2 & 47 & $\mathrm{M}$ & $\mathrm{N}$ & + & + \\
\hline 3 & 40 & $\mathrm{~F}$ & $\mathrm{~N}$ & + & + \\
\hline 4 & 35 & $\mathrm{M}$ & $\mathrm{Y}$ & + & + \\
\hline 5 & 30 & $\mathrm{M}$ & $\mathrm{N}$ & + & + \\
\hline 6 & 37 & $\mathrm{M}$ & $\mathrm{N}$ & + & - \\
\hline 7 & 35 & $\mathrm{~F}$ & $\mathrm{~N}$ & + & + \\
\hline 8 & 53 & $\mathrm{M}$ & $\mathrm{N}$ & - & + \\
\hline 9 & 45 & $\mathrm{~F}$ & $\mathrm{~N}$ & + & + \\
\hline 10 & 30 & $\mathrm{~F}$ & $\mathrm{Y}$ & + & + \\
\hline 11 & 33 & $\mathrm{M}$ & $\mathrm{N}$ & + & + \\
\hline 12 & 35 & $\mathrm{M}$ & $\mathrm{N}$ & + & + \\
\hline 13 & 54 & $\mathrm{~F}$ & $\mathrm{~N}$ & + & + \\
\hline 14 & 28 & $\mathrm{M}$ & $\mathrm{N}$ & - & + \\
\hline 15 & 36 & $\mathrm{~F}$ & $\mathrm{~N}$ & - & + \\
\hline & & & & + & + \\
\hline & & & & + & + \\
\hline & 35 & & + & + \\
\hline
\end{tabular}

$38.3 \pm 17.3$ years) were found to show positive results, and were thus diagnosed as LAR (Tables 1 and 2); with 11/12 of these patients were part of the 14 rhinitis patients, and ranged from 30 years to 54 years in age. Thus, 11 patients were diagnosed as LAR by both NAPT and local slgE (Figure 1). The sensitivity, specificity, PPV, NPV, and DA for local slgE as a diagnostic tool

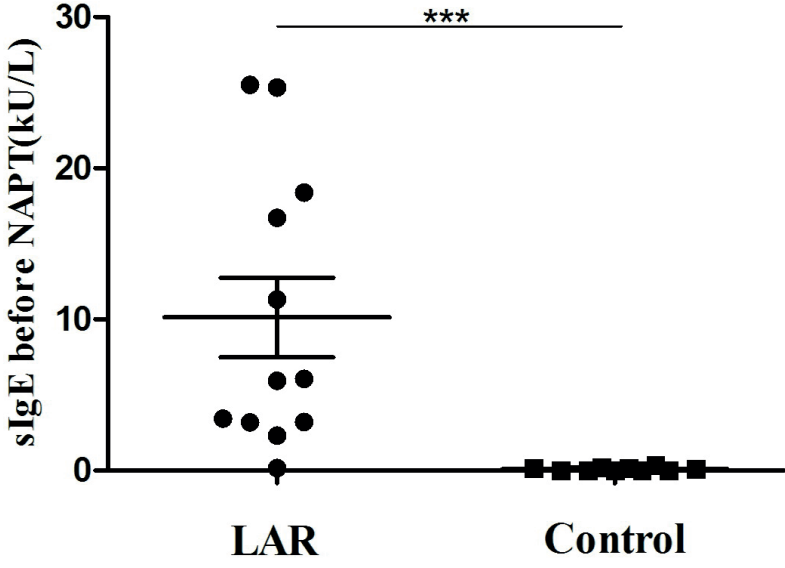

Figure 2. Measurement of specific Immunoglobulin E (slgE) before nasal allergen provocation test (NAPT)

for the diagnosis of LAR were $91.7 \%(95 \% \mathrm{Cl}, 64.6 \%$ to $98.5 \%)$, $95.1 \%$ ( $95 \% \mathrm{Cl}, 86.5 \%$ to $98.3 \%)$, $78.6 \%$ (95\% Cl, $52.4 \%$ to $92.4 \%)$, $98.3 \%$ ( $95 \% \mathrm{Cl}, 91.0 \%$ to $99.7 \%)$, and $94.5 \%(95 \% \mathrm{Cl}, 86.7 \%$ to $97.9 \%)$, respectively.

To verify the diagnosis of LAR, clinical markers and laboratory biomarkers were also measured following NAPT. Table 2 shows the demographic characteristics, nasal symptoms VAS scores, nasal airway resistance and the level of local slgE before and after NAPT of patients diagnosed as LAR. The local slgE level was significantly higher in the LAR group than the healthy control group before NAPT (Figure 2). Among these patients, one patient (number 12; italicized) had a positive NAPT result,

Table 2. The Demographic characteristics and VAS, nasal airway resistance and local slgE level at baseline and after NAPT of LAR patients.

\begin{tabular}{|c|c|c|c|c|c|c|c|c|c|}
\hline \multirow[t]{2}{*}{ No. } & \multirow[t]{2}{*}{ Age } & \multirow[t]{2}{*}{ Gender } & \multirow[t]{2}{*}{ Asthma } & \multicolumn{2}{|c|}{ VAS } & \multicolumn{2}{|c|}{ NAR } & \multicolumn{2}{|c|}{ Local slgE } \\
\hline & & & & Before & After & Before & After & Before & After \\
\hline 1 & 38 & $M$ & $\mathrm{~N}$ & 20 & 25 & 2.203 & 8.005 & 11.29 & 33.72 \\
\hline 2 & 46 & $M$ & $\mathrm{~N}$ & 10 & 30 & 0.377 & 2.394 & 3.21 & 6.96 \\
\hline 3 & 41 & $\mathrm{~F}$ & $\mathrm{~N}$ & 22 & 40 & 0.599 & 1.184 & 2.32 & 5.46 \\
\hline 4 & 34 & $M$ & $\mathrm{Y}$ & 15 & 25 & 0.522 & 5.264 & 16.75 & 26.31 \\
\hline 5 & 30 & $M$ & $\mathrm{~N}$ & 16 & 40 & 0.279 & 0.960 & 25.54 & 30.22 \\
\hline 6 & 36 & $\mathrm{~F}$ & $\mathrm{~N}$ & 14 & 14 & 0.806 & 1.279 & 5.96 & 29.51 \\
\hline 7 & 55 & $\mathrm{~F}$ & $\mathrm{~N}$ & 18 & 40 & 1.530 & 6.329 & 18.41 & 33.52 \\
\hline 8 & 46 & $\mathrm{~F}$ & $\mathrm{~N}$ & 18 & 22 & 0.322 & 2.021 & 25.35 & 25.96 \\
\hline 9 & 31 & $\mathrm{~F}$ & $Y$ & 21 & 27 & 1.373 & 2.070 & 3.22 & 8.25 \\
\hline 10 & 33 & $M$ & $\mathrm{~N}$ & 21 & 34 & 0.687 & 1.264 & 3.66 & 9.45 \\
\hline 11 & 35 & $M$ & $\mathrm{~N}$ & 15 & 26 & 0.733 & 1.178 & 6.17 & 15.31 \\
\hline 12 & 36 & $M$ & $N$ & 16 & 25 & 0.801 & 1.278 & 0.17 & 0.31 \\
\hline
\end{tabular}

$\mathrm{N}=$ no; $\mathrm{Y}=$ yes; $\mathrm{M}=$ male; $\mathrm{F}=$ female; VAS = visual analogue scale; Der $\mathrm{f}=$ Dermatophagoides farinae; NAPT = nasal allergen provocation test; $\mathrm{NAR}=$ nasal airway resistance 
A

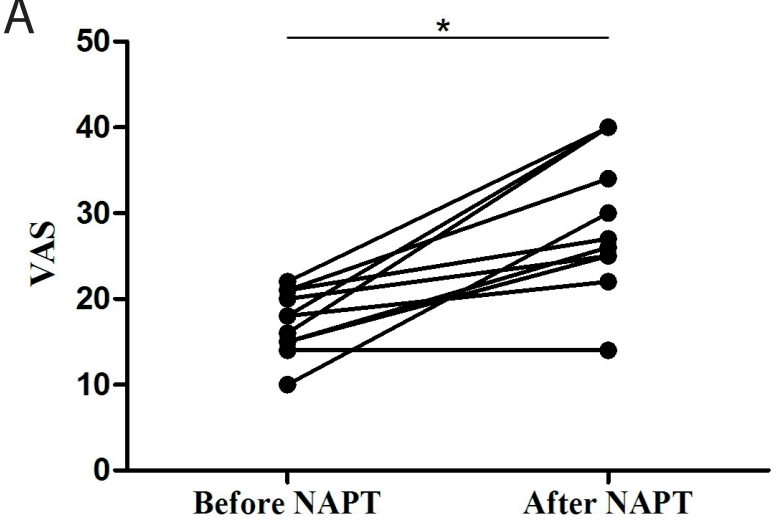

C

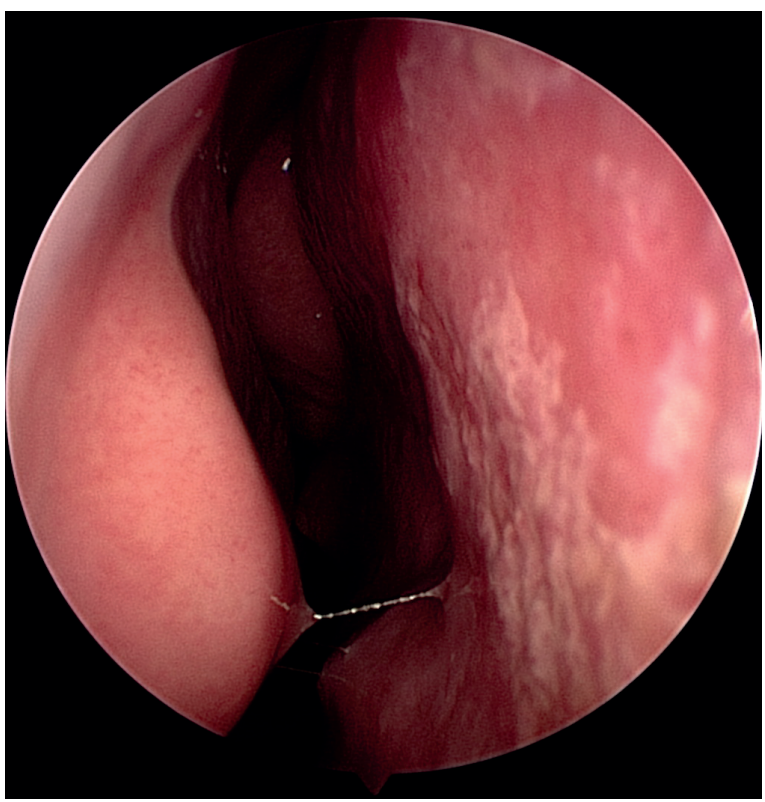

B

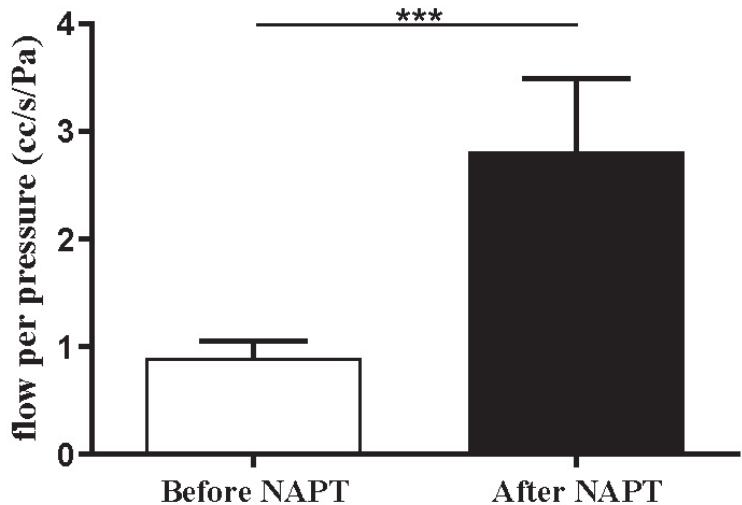

$\mathrm{D}$

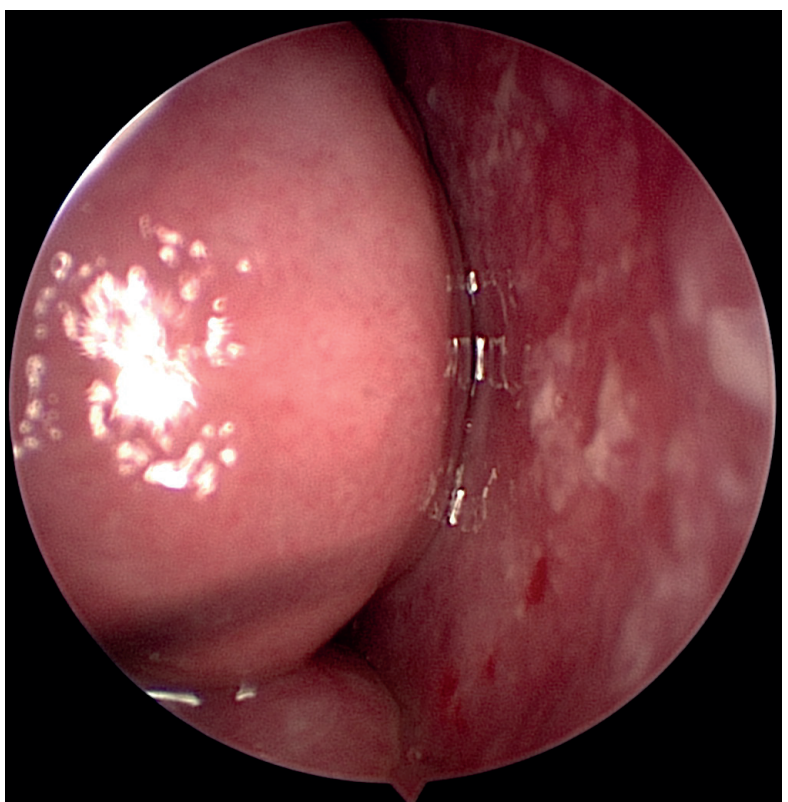

Figure 3. Effect of nasal allergen provocation test (NAPT) in patients with local allergic rhinitis (LAR). NAPT significantly increased the symptom score (A) and nasal airway resistance (NAR) (B); and led swelling of the inferior turbinate and production of large quantities of watery secretions in the nasal cavity (representative endoscopic images of the nasal cavity before (C) and after (D) NAPT).

but a negative local slgE result. Overall, the VAS scores and NAR in the LAR group were significantly increased from baseline after NAPT (Figures 3A and 3B). The diluent saline solution did not significantly alter the resting NAR $(\mathrm{cc} / \mathrm{s} / \mathrm{Pa})$ in these patients (before saline $=20$ vs after saline $=20$ ). Endoscopic examination demonstrated that NAPT also increased swelling of the inferior turbinate and watery secretion in the nasal cavity in these patients (Figures 3C and 3D). Similarly, assessment of inflammatory mediators in nasal secretions demonstrated that the levels of local slgE, ECP, histamine and leukotriene $C 4$ were also significantly increased from baseline after NAPT in LAR patients (Figure 4).

\section{Discussion}

Chronic rhinitis continues to be a significant problem that decreases the quality of life of affected individuals ${ }^{(1)}$. As a specific phenotype of chronic rhinitis, LAR has been widely discussed in the recent years ${ }^{(2,8,9-14)}$. Furthermore, according to the currently employed diagnostic criteria of LAR, many patients previously diagnosed as non-allergic rhinitis patients should be given a diagnosis of LAR ${ }^{(12)}$. With the development of NAPT, increasingly more patients have been diagnosed as LAR; with this endotype affecting $25.7 \%$ of the rhinitis population and including about half of the patients previously diagnosed with nonallergic rhinitis ${ }^{(10)}$.

For the diagnosis of LAR, neither skin prick testing nor determination of the presence of serum slgE antibodies is thought to be useful, and a NAPT is needed to identify the sensitizing allergen or allergens ${ }^{(11)}$. However, NAPT is not available in every clinic, and is also somewhat limited as it is difficult to apply in children and is time consuming ${ }^{(23)}$. In contrast, nasal secretion is easy to obtain, and contains a variety of inflammatory cells, mediators, 
A

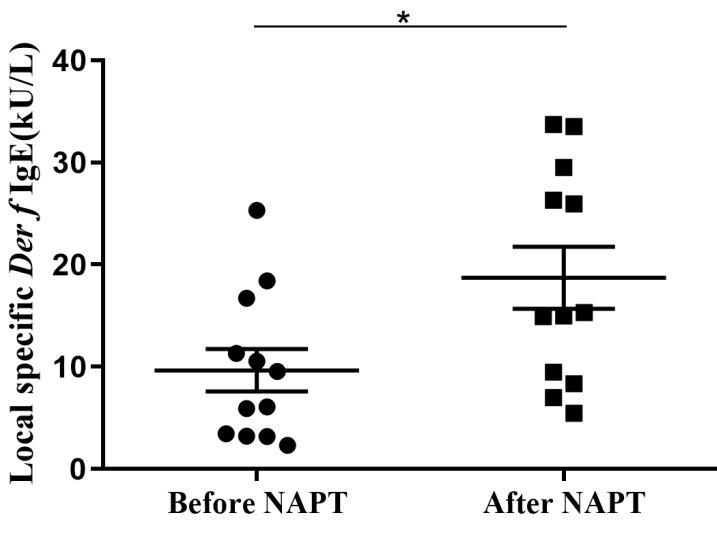

C

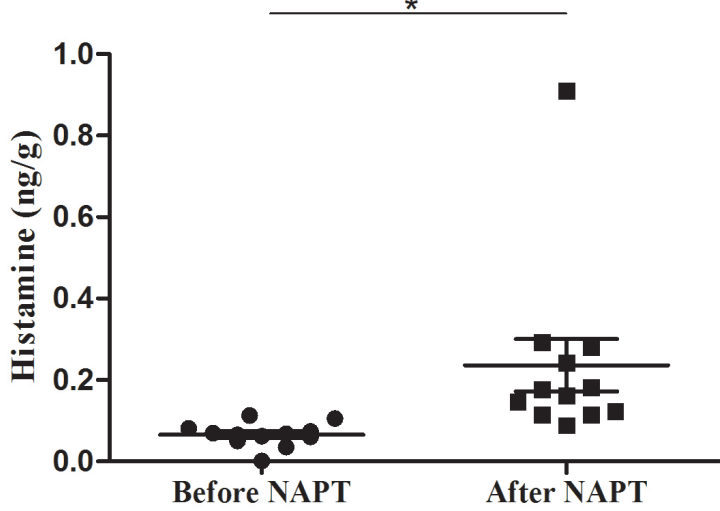

B

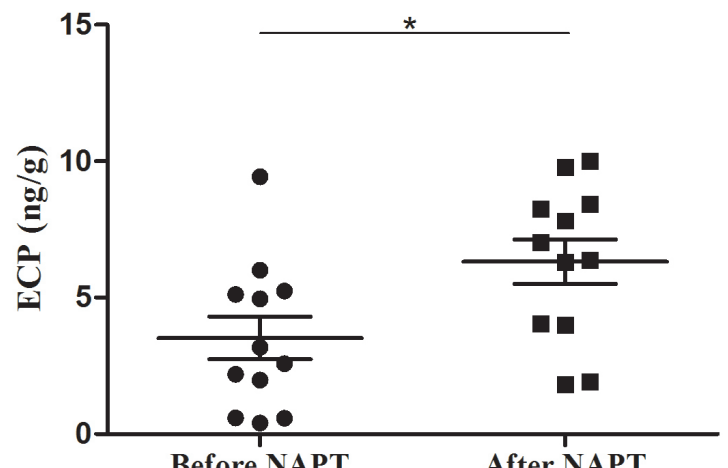

D

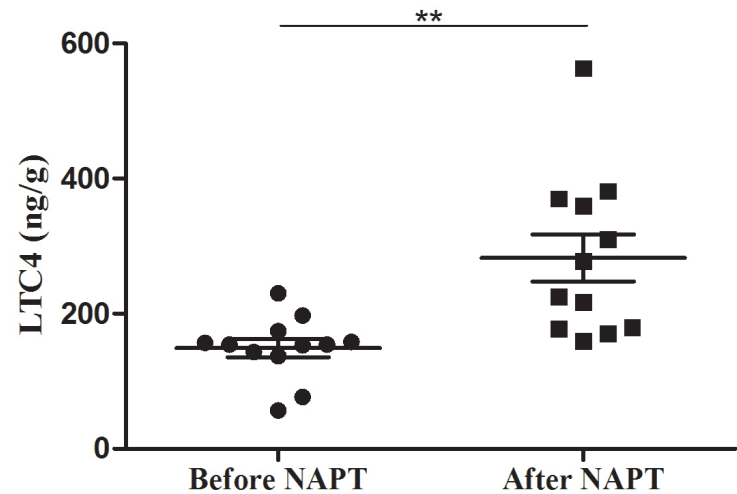

Figure 4. Measurement of Der $f$-specific Immunoglobulin $E$ (Der $f$-slgE) and inflammatory mediators before and after nasal allergen provocation test (NAPT). A, Derf-slgE; B, eosinophil cationic protein (ECP); C, histamine; D, leukotriene C4 (LTC4).

and other immunologic markers. Several studies have shown that local specific IgE is synthesized in the nasal mucosa of rhinitis patients ${ }^{(7,8)}$, and findings from a previous study of ours has suggested that nasal secretion could be used for diagnosis of AR (18). Therefore, for the current study we hypothesized that local slgE in nasal secretion could be used for the diagnosis of LAR. In this study, only rhinitis patients who had negative serum slgE results and positive results of local eosinophils were enrolled to rule out any systemic allergic reactions, and the incidence of LAR was verified by the presence of local slgE and the effect of NAPT with Dermatophagoides farinae (Der $f$ ) and mugwort. Using these diagnostic criteria, our study demonstrated that only around $20 \%$ (14/73) of the study cohort comprised LAR patients, of whom the $78.6 \%$ (11/14 patients) had positive NAPT results for Derf. This result was interesting because none of them was allergic to mugwort. This might be due to these patients were recruited from February to May and mugwort is the main aeroallergen in Autumn in Beijing (duration from late July to end of September) ${ }^{(24)}$. One patient demonstrated a positive NAPT reaction, but a negative local slgE result. Despite the negative result for local slgE, this patient would be diagnosed as LAR according to the earlier studies ${ }^{(11-14)}$. Similarly, two patients who had a positive result for low levels of local slgE, but negative NAPT results. Based on classification criteria of Rondon and colleagues ${ }^{(11)}$, these two patients could not be diagnosed as LAR, because they did not meet all three requirements of 1) local production of $\lg \mathrm{E}$, 2) Th2 inflammatory pattern in nasal secretion when exposed to sensitizing allergens, and 3) positive results of nasal allergen provocation test (NAPT) without systemic atopy, including increased level of slgE, tryptase and eosinophil cationic protein $(E C P)$ in the nasal secretion. Collectively, these findings indicate that the diagnostic accuracy of local slgE for LAR was $94.5 \%$. There was an earlier report that local slgE also could be detected in nonallergic rhinitis patients and healthy subjects at baseline (25). In the present study, there were three rhinitis patients had positive slgE but negative NAPT results (Table 1) and this was in accordance with the former study ${ }^{(25)}$. However, none of the healthy control reached diagnostic threshold in our study despite 5 of them had slgE results lower than $0.35 \mathrm{kU} / \mathrm{L}$ (The slgE results of the rest 6 healthy control were 0 ). This was might be due to the different diagnostic kits in these two studies. In the current study, all the patients with LAR had a positive rapid response; and none suffered a late response (data not show). These findings are in accordance with those of Rondon and colleagues ${ }^{(15)}$ and support the existence of a persistent local synthesis of slgE in nasal mucosa of patients with LAR that rapidly enhances after nasal exposure to aeroallergens ${ }^{(9)}$. Furthermore, $E C P$ has been shown as one of the indicators of $L A R^{(10)}$. In the 
present study, we evaluated several mediators, besides ECP, as markers of LAR; and found that the levels of ECP and leukotriene C4 were significantly increased after NAPT in the patients diagnosed as LAR. This is in accordance with findings from other studies ${ }^{(12,26)}$ and indicative of LAR being a Th2 driven nasal mucosa inflammation, as both ECP and leukotriene C4 are Th2-mediated inflammatory markers ${ }^{(26)}$. Although histamine is not a classic Th2 inflammatory marker, it has been shown to be a main mediator during the rapid early phase reaction of rhinitis ${ }^{(27)}$. In this study, 12 patients had significantly higher levels of histamine compared to baseline levels following stimulation with allergen solution. We also observed that the patients had the classical symptoms of nasal irritation, which corresponds to the high level of histamine. For the remaining 60 patients in the cohort, even though they had negative serum slgE results and positive local eosinophils results, these patients did not have positive local slgE findings. Thus, according to the former diagnostic criteria ${ }^{(27-29)}$, these patients could be diagnosed as non-allergic rhinitis with eosinophilic syndrome (NARES) patients. However, this needs to be confirmed in further investigations.

The findings of the present study are somewhat limited because of the relatively small number of patients. An earlier study in 2014 reported that LAR affects $25.7 \%$ of the rhinitis population (10). This prevalence of LAR is quite high, compared to the present cohort, in which only $12 / 212$ patients (5\%) met the criteria of LAR during screening. This might be explained by the ethnic differences of the cohorts because most previous studies of LAR to date have been performed in Caucasians in Europe.

\section{Conclusion}

In conclusion, the present study suggested that measurement of local slgE levels in nasal secretion, instead of NAPT, is a reliable and effective clinical method for a diagnosis LAR. However, these results need to be confirmed in a much larger cohort in a multicenter study and in multi-ethnic patients.

\section{Acknowledgements}

This work was supported by grants from National Key R \& D Program of China (2016YFC20160905200), the program for Changjiang Scholars and Innovative Research Team (IRT13082); National Science Fund for the Major International Joint Research Program (81420108009); National Natural Science Foundation of China (81630023, 81100704, 81441029, 81441031, 81570894, 81400444, and 81470678); Capital Health Development Foundation (2016-1-2052).

\section{Authorship contribution}

YM prepared the manuscript and performed the statistical analysis. YW, HL, KW and NM collected the clinical data and samples. CW participated in collection of the clinical data and samples, and was involved in the preparation of the manuscript. LZ designed the study and participated in the interpretation and discussion of the data.

\section{Conflict of interest}

No conflict of interest.

\section{References}

1. Bousquet J, Khaltaev N, Cruz AA, Denburg J, Fokkens WJ, Togias A, et al. Allergic Rhinitis and its Impact on Asthma (ARIA) 2008. Allergy 2008;63(suppl 86):8-160.

2. Rondon C, Canto G, Blanca M. Local allergic rhinitis: a new entity, characterization and further studies. Curr OpinAllergy Clin Immunol 2010, 10:1-7.

3. Hellings PW, Klimek L, Cingi C, Agache I, Akdis C, Bachert C, et al. Non-allergic rhinitis: Position paper of the European Academy of Allergy and Clinical Immunology. Allergy. 2017;72(11):1657-65.

4. Ledford D. Inadequate diagnosis of nonallergic rhinitis: assessing the damage. Allergy Asthma Proc 2003; 24:155-162.

5. Hens G, Vanaudenaerde BM, Bullens DM, Piessens M, Decramer M, Dupont $L$, et al. Sinonasal pathology in nonallergic asthma and COPD: 'united airway disease' beyond the scope of allergy. Allergy 2008; 63:261267.

6. Meltzer EO, Blaiss MS, Naclerio RM, Stoloff SW, Derebery MJ, Nelson HS, et al. Burden of allergic rhinitis: allergies in America, Latin America, and Asia-Pacific adult surveys. Allergy Asthma Proc 2012; 33:113-141.
7. Huggins KG, Brostoff J. Local production of specific IgE antibodies in allergic-rhinitis patients with negative skin tests. Lancet 1975; 2:148-50.

8. Powe DG, Jagger C, Kleinjanw A, Carney AS, Jenkins D, Jones NS. 'Entopy': localized mucosal allergic disease in the absence of systemic responses for atopy. Clin Exp Allergy 2003;33:1374-1379.

9. Rondon C, Fernàndez J, López S, Campo P, Doña I, Torres MJ, et al. Nasal inflammatory mediators and specific IgE production after nasal challenge with grass pollen in local allergic rhinitis. J Allergy Clin Immunol 2009, 124:1005-1011.

10. Rondon C, Campo P, Zambonino MA, Blanca-Lopez N, Torres MJ, Melendez L, et al. Follow-up study in local allergic rhinitis shows a consistent entity not evolving to systemic allergic rhinitis. J Allergy Clin Immunol 2014; 133:1026-31.

11. Rondon C, Campo P, Togias A, Fokkens WJ, Durham SR, Powe DG, et al. Local allergic rhinitis: Concept, pathophysiology, and management. J Allergy Clin Immunol 2012; 129:1460-7.

12. Rondon C, Campo P, Herrera R, BlancaLopez N, Melendez L, Canto G, et al. Nasal allergen provocation test with multiple aeroallergens detects polysensitization in local allergic rhinitis. J Allergy Clin Immunol 2011; 128:1192-7.

13. Rondon C, Romero JJ, Lopez S, Antunez C, Martin-Casanez E, Torres MJ, et al. Local lgE production and positive nasal provocation test in patients with persistent nonallergic rhinitis. J Allergy Clin Immunol 2007; 119:899-905.

14. Rondon C, Campo P, Eguiluz-Gracia I, Plaza C, Bogas G, Galindo P, Mayorga C, Torres MJ. Local allergic rhinitis is an independent rhinitis phenotype: The results of a 10-year follow-up study. Allergy. 2018;73(2):470-478

15. Rondon C, Dona I, Lopez S, Campo P, Romero JJ, Torres MJ, et al. Seasonal idiopathic rhinitis with local inflammatory response and specific IgE in absence of systemic response. Allergy 2008;63: 1352-1358.

16. Peebles RS, Hartert TV. In vivo diagnostic procedures: skin testing, nasal provocation, and bronchial provocation. Methods 1997; 13:14-24.

17. Melillo G, Bonini S, Cocco G, Davies RJ, de Monchy JG, Frølund L, et al. Provocation tests with allergens. Report prepared by the European Academy of Allergology and 
Clinical Immunology Subcommittee on provocation tests with allergens. Allergy 1997;52(Suppl):5-36.

18. Meng $Y$, Lou $H$, Wang $Y$, Wang $C$, Zhang $L$. The use of specific immunoglobulin e in nasal secretions for the diagnosis of allergic rhinitis. Laryngoscope. 2018 Sep;128(9):E311-E315.

19. Howarth PH, Persson CGA, Meltzer EO, Jacobson MR, Durham SR, Silkoff PE, et al. Objective monitoring of nasal airway inflammation in rhinitis. J Allergy Clin Immunol 2005;115: S414-41.

20. Watelet JB, Gevaert P, Holtappels G, van Cauwenberge $\mathrm{P}$, Bachert $\mathrm{C}$. Collection of nasal secretions for immunological analysis. Eur Arch Otorhinolaryngol 2004; 261: 242-246.

21. Augé J, Vent J, Agache I, Airaksinen L, Campo Mozo P, Chaker A, et. al. EAAC Position paper on the standardization of nasal allergen challenges. Allergy 2018;73: 1597-1608.

22. Gosepath J, Amedee RG, Mann WJ. Nasal provocation testing as an international standard for evaluation of allergic and nonallergic rhinitis. Laryngoscope 2005; 115:512-516.

23. Colavita L, Catalano N, Sposito G, Loddo S,
Galletti B, Salpietro C, et al. Local Allergic Rhinitis in Pediatric Patients: Is lgE Dosage in Nasal Lavage Fluid a Useful Diagnostic Method in Children? Int J Mol Cell Med 2017 ;6 (3): 174-182

24. Lou H, Ma S, Zhao Y, Cao F, He F, Liu Z, et al. Sensitization patterns and minimum screening panels for aeroallergens in selfreported allergic rhinitis in China. Sci Rep 7: 9286, 2017.

25. Matteo G, Antonio V. N. G, Lucia I, Vitaliano NQ, Nicola Q, Massimo L, et al. Local allergic rhinitis: entopy or spontaneous response? World Allergy Organ J. 2016; 9;39:1-6.

26. Marcucci F, Sensi LG, Migali E, Coniglio G. Eosinophil cationic protein and specific lgE in serum and nasal mucosa of patients with grass-pollen-allergic rhinitis and asthma. Allergy 2001; 56:231-236.

27. Church MK. Allergy, Histamine and Antihistamines. Handb Exp Pharmacol 2017; 241:321-331.

28. Leone C, Teodoro C, Pelucchi A, Mastropasqua B, Cavigioli G, Marazzini L, et al. Bronchial responsiveness and airway inflammation in patients with nonaller gic rhinitis with eosinophilia syndrome. Allergy Clin Immunol 1997; 100:775-780.

29. Zambetti G, Ciofalo A, Romeo R, Soldo P,
Fusconi M, Greco A, et al. Nasal histamine responses in nonallergic rhinitis with eosinophilic syndrome. Allergy Rhinol 2015; 6: e94-e100.

Chengshuo Wang, MD, PhD

Department of Otolaryngology Head and Neck Surgery

Beijing TongRen Hospital

Capital Medical University

No. 1, Dongjiaominxiang

Dongcheng District

Beijing 100730, China

E-mail:wangcs830@126.com

Luo Zhang, MD, PhD

Beijing Institute of Otolaryngology

No. 17, HouGouHuTong

DongCheng District

Beijing, 100005; China

E-mail: dr.luozhang@139.com 